\title{
ASH 2013: constant progress: not only for B-cell neoplasms
}

\author{
Michael Steurer
}

Received: 21 August 2014 / Accepted: 26 August 2014 / Published online: 16 September 2014 (C) Springer-Verlag Wien 2014

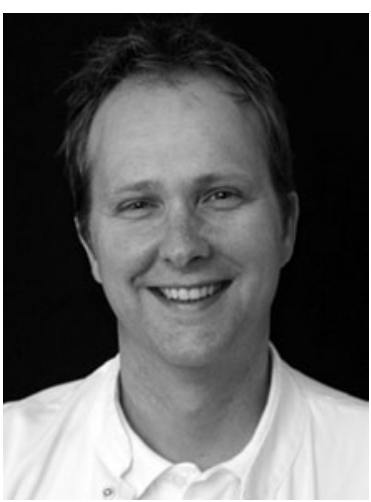

Michael Steurer
The 55th Annual Meeting of the American Society of Hematology (ASH) held in New Orleans was attended by 21,000 participants from over 110 countries. Despite the famous meeting place ("The Big Easy"), selecting the most relevant data out of a wealth of $>5000$ abstracts and $>3000$ presentations represents a major challenge. Therefore, in this issue of MEMO several experts in the field have worked through the wealth of data presented to extract the most meaningful clinical and translational data for the readers of this journal. Moreover, I would like to congratulate all of them for their excellent performance.

As already covered in past post-ASH issues of MEMO, the probably most practice-changing developments are taking place in the field of chronic lymphocytic leukemia (CLL) and non-Hodgkin's lymphoma. The introduction of B-cell receptor (BCR) inhibitors, such as ibrutinib or idelalisib has enabled us to achieve clinically relevant results even in high-risk and otherwise refractory patients. Whereas in the last 2 years mainly phase II studies (exploring BCR inhibitors given as single agent) were presented, we can now base our clinical decisions on phase III trials [1]. Moreover, the first results from combination trials with immunochemotherapy have been discussed. Nevertheless, a pleasantly great number of randomized trials demonstrate that immunochemotherapy is an important mainstay for CLL treatment, in particular when novel, potent anti-CD20 antibodies such as obinutuzumab (GA-101) are used. Another promising drug presented at ASH 2013 is the Bcl-2 inhibitor ABT-199, which has shown to be a very effective drug for

M. Steurer, MD $(\bowtie)$

Laboratory for Molecular Genetics \& Diagnostics,

Department of Internal Medicine V, Innsbruck Medical University,

Anichstr. 35,

6020 Innsbruck, Austria

e-mail: michael.steurer@i-med.ac.at high-risk relapsed/refractory CLL. Altogether, progress for CLL and indolent lymphoma treatment is being made with giant steps-thanks to critical contributions of basic researchers and translational scientists.

However, as reviewed by both Dr. Burgstaller [2] and Dr. Nösslinger [3] the translation of novel biologic and/ or genetic findings into successful clinical management remains difficult. For example, molecular genetic studies have led to the prognostically relevant distinction between germinal-center (GC) and non-GC type diffuse large B-cell lymphomas (DLBCL) or the identification of so-called double lymphomas. However, so far this knowledge has not led to the development of novel and/ or targeted therapeutic strategies. Dr. Nösslinger discusses whether the adjunct of lenalidomide may be able to improve the outcome for patients with non-GC-type DLBCL but this encouraging finding needs validation in larger trials. Meanwhile, the current focus of clinical research concerning aggressive non-Hodgkin's lymphomas remains the optimization of conventional chemotherapy regimens.

As Dr. Burgstaller discusses [2], new genetic techniques such as massive parallel sequencing have been able to determine the prevalence of several recurrent mutations in genes such as TET2, SF3B1, ASXL1, SRSF2, DNMT3A, and RUNX1 in myelodysplastic syndromes (MDS). However, the clinical heterogeneity of MDS is also reproducible on the molecular level showing a wide range of mutations that rarely exceed a prevalence of $10 \%$. Hence, clinical decision making still relies on clinical and conventional cytogenetic findings as reflected, for example, in the (R-) IPSS system. However, first clinical data on the use of the novel nucleoside analog sapacitabine appears promising, in particular for patients failing demethylating agents. In summary, if we want to achieve further progress-not only concerning MDS and aggressive lymphomas-further (independent!) basic research (and its 
funding!) is needed to identify new potentially druggable targets and therapeutic strategies.

With the discovery of the activating JAK2V617F mutation and the subsequent development of JAK-inhibitors, major progress has been made for patients suffering from Philadelphia-chromosome negative myeloproliferative neoplasms (MPN). As reported by Dr. Schmidt [4], the discovery of a new mutational hotspot in the Calreticulin (CALR) gene is another important step forward in MPN research. As these mutations seem to occur almost mutually exclusive from JAK2V617F mutations assessment of CALR mutations allows a significant improvement of the diagnostic accuracy of molecular genetic analyses. Incorporating CALR mutational screening allows the identification of a clonal genetic marker in $\sim 85 \%$ of patients with essential thrombocythemia and $\sim 90 \%$ of patients with primary myelofibrosis, respectively. The clinical consequences of the presence of CALR mutations, however, are still under investigation.

As introduced by Dr. Feistritzer's and Dr. Wildner's elegant article concerning the most important hemostaseologic findings, ASH has recently established the Choosing Wisely ${ }^{\circledR}$ campaign [5]. The ASH-Choosing Wisely ${ }^{\circledR}$ Task Force was established to identify hematologic tests and treatments that are of uncertain clinical value, thereby increasing costs and exposing the patients to potential risks. Remarkably, three of the five tests and treatments identified were in the field of coagulation reflecting that in particular subspecialty of hematology more evidencebased treatment guidelines are necessary. The recent findings regarding age-adjusted D-dimer levels in elderly patients are very convincing and should be readily incorporated into current treatment algorithms. Further recommendation from the ASH-Choosing Wisely ${ }^{\circledR}$ Task Force for practising hematologists are eagerly awaitednot only concerning hemostaseology.

I hope this issue of $M E M O$ transfers not only valuable updates for routine clinical practice to its readers but also excitement, curiosity, and motivation for further support of translational as well as clinical research and clinical trials to improve future patient care.

\section{Conflict of interest}

The author has no conflicts of interest to disclose.

\section{References}

1. Steurer M. ASH Update 2013: chronic lymphocytic leukemia and indolent lymphoma. Memo 2014;3. DOI 10.1007/ s12254-014-0174-4.

2. Burgstaller S. Myelodysplastic syndromes (MDS). Memo 2014;3. DOI 10.1007/s12254-014-0158-4.

3. Nösslinger T. Aggressive lymphoma at ASH 2013: R-CHOP under attack? Memo 2014;3. DOI 10.1007/ s12254-014-0148-6.

4. Schmidt S. ASH 2013: short review on myeloproliferative neoplasms. Memo 2014;3. DOI 10.1007/s12254-014-0168-2.

5. Feistritzer C, Wildner SM. Highlights of the American Society of Hematology Meeting 2013: hemostaseology. Memo 2014;3. DOI 10.1007/s12254-014-0157-5. 